\title{
Prevalência de sobrepeso e obesidade em homens adultos segundo dois critérios de diagnóstico antropométrico
}

\author{
Ronaldo Domingues Filardo', Edio Luiz Petroski ${ }^{2}$ \\ 1 Universidade Federal do Paraná - Departamento de Educação Física \\ GPAQ (Grupo de Pesquisa em Atividade Física e Qualidade de Vida - PUCPR). \\ ${ }^{2}$ Universidade Federal de Santa Catarina - Campus Universitário - Trindade - Caixa Postal 476 \\ Centro de Desportos - Núcleo de Pesquisa em Cineantropometria e Desempenho Humano.
}

Filardo, R. S.; Petroski, E.L; Prevalência de sobrepeso

e obesidade em homens adultos segundo dois critérios de diagnóstico antropométrico.

Motricidade 3(4): 46-54, 2007

\section{Resumo}

Verificar e comparar a prevalência de sobrepeso e obesidade por dois critérios de diagnóstico antropométrico em homens, entre $20 \mathrm{e}$ 40 anos de idade. Participaram do estudo, 400 sujeitos de 20 a 40 anos de idade iniciantes de programas de exercício físico de academias de Curitiba-PR, divididos em 4 faixas etárias. Os diagnósticos antropométricos de sobrepeso e obesidade foram: IMC e $\% \mathrm{G}$, segundo os pontos de corte sugeridos pela OMS (2000) e Lohman (1992), respectivamente. Para tanto, foram mensurados: peso, estatura e quatro dobras cutâneas (tríceps, subescapular, supra-ilíaca e panturrilha medial). A prevalência de excesso de peso foi significantemente $(p<0,05)$ maior no diagnóstico antropométrico de $\% \mathrm{G}(66,2 \%)$ que no de IMC (45,6\%), sendo $53,2 \%$ para sobrepeso e $13 \%$ para obesidade. No diagnóstico de IMC, a prevalência de sobrepeso e obesidade foi 36,3\% e $9,3 \%$, respectivamente. Segundo o diagnóstico de $\% \mathrm{G}$, um sujeito, entre 35-39 anos, tem 300\% maior prevalência de obesidade e $45 \%$ de sobrepeso que um sujeito de 20-24 anos de idade. Da mesma forma, no diagnóstico por IMC, um sujeito na faixa etária de 30-34 anos com sobrepeso a prevalência é de $46 \%$ maior que outro sujeito da faixa etária de 20-24 anos. O estudo encontrou alta prevalência de excesso de peso (um a cada três sujeitos) ao se inscreverem em academia de ginástica. $\mathrm{O}$ risco de obesidade pode se melhor identificado quando o excesso de peso é diagnosticado através do \%G.

Palavras Chave: obesidade, sobrepeso, IMC, gordura, masculino.

\section{Abstract}

Adult overweight and obesity prevalence by two anthropometric criteria

To determine and compare overweight and obesity by two anthropometric criteria, BMI and $\%$ Fat, of adults aged 20 to 40 years old. Data were collected from 400 subjects enrolled on distinct exercise training programs in Curitiba, Paraná. Subjects were organized in 4 age groups from age 20 to 40 years old. BMI and \%Fat overweight and obesity criteria were those of WHO (2000) and Lohman (1992), respectively. For such, weight, stature and fours skin folds (triceps, subescapula, iliac crest and medial calf) data were collected. The excess of body weight prevalence was significantly higher $(\mathrm{p}<0.05)$ for $\%$ Fat $(66.2 \%)$ than for BMI $(45.6 \%)$ of which $53.2 \%$ were overweight and $13 \%$ obese. For BMI, the prevalence of overweight and obesity were, respectively, $36.3 \%$ and $9.3 \%$. Accordingly to the $\%$ Fat diagnostic, a subject between ages 35-39 years old has a $300 \%$ and $45 \%$ chance of being classified as obese and overweight, respectively, than a subject aged from $20-24$ years old. Likewise, for BMI, a subject has a $46 \%$ chance of being classified as overweight at the age $30-34$ years old than a subject between ages 20-24 years old. This study found a prevalence of excess body weight for one out of three at the moment of enrollment. The risk of obesity, as excess of body weight, can better be identified by $\% \mathrm{~F}$ than BMI.

Keywords: overweigh, obesity, BMI, Fat, male. 


\section{Introdução}

A prevalência de excesso de peso e de obesidade tem aumentado em adultos brasileiros durante as últimas décadas. Com o avanço da idade, ocorre o aumento de massa e gordura corporal e, como consequência, o desenvolvimento do sobrepeso e/ou obesidade. Estas condições constituem um importante problema de saúde pública, pelo fato de estes incrementarem o risco para inúmeras doenças e desordens ${ }^{13,20}$. A Organização Mundial de Saúde (OMS) ${ }^{29}$ estima que a obesidade atingiu proporções epidêmicas globais, com mais de 1 bilhão de adultos com sobrepeso e estima-se que no mínimo 300 milhões são clinicamente obesos. Contudo, pode coexistir com subnutrição em países em desenvolvimento. Assim, a obesidade é uma condição complexa com sérias dimensões psicológicas e sociais - que afeta todas as idades e grupos sócio-econômicos. O Brasil, em transição nutricional, passa rapidamente de um problema de déficit dietético para um de excesso ${ }^{2}$.

Comumente, utiliza-se para classificação da obesidade o Índice de Massa Corporal $\left(\mathrm{IMC}=\mathrm{kg} / \mathrm{m}^{2}\right)$, pela sua facilidade de utilização e pela boa relação com a gordura corporal na população geral ${ }^{14}$, pois somente os valores de Massa Corporal $(\mathrm{MC}=\mathrm{kg}$ ) e estatura (EST= $=\mathrm{kg} / \mathrm{m}^{2}$ ) são necessários para o seu estabelecimento. Além disso, o que faz com que este índice tenha uma grande aceitação pela comunidade científica é o seu relacionamento com o desenvolvimento de desordens metabólicas e inúmeras doenças ${ }^{3}$; mas, isoladamente, esse índice não representa o principal indicador para o aumento das taxas de mortalidade 9

Segundo o sistema de classificação do $\mathrm{IMC}^{30}$, considera-se pessoa obesa aquela que possui um IMC maior ou igual a $30 \mathrm{~kg} / \mathrm{m}^{2}$; com sobrepeso aquela com IMC entre 25 e $29,9 \mathrm{~kg} / \mathrm{m}^{2}$. Contudo, a interpretação do IMC necessita de atenção redobrada, pois esse índice não distingue entre as massas de gordura e muscular, o que pode ocasionar significativos $\operatorname{erros}^{10,13}$; como, por exemplo, o idêntico IMC de um halterofilista e o de uma mulher obesa de meia-idade. Quando se desconsidera esses óbvios extremos, existe uma boa correlação entre o IMC e o percentual de gordura corporal em uma população geral ${ }^{25}$.

Com o passar dos anos, as recomendações em relação aos critérios de classificação, baseados em distribuições de populações observadas, foram sendo modificadas, pois o ponto de corte ou faixa adotada é outro cuidado a ser tomado na adoção de um critério. A diferença entre os valores adotados pode influenciar a prevalência de indivíduos a serem classificados, pois a mudança no ponto de corte de 27,8 para $25 \mathrm{~kg} / \mathrm{m}^{2}$ no IMC altera a prevalência de homens americanos com sobrepeso de 33,3\% para 59,4\%, o que indica que a determinação de critérios pode influenciar na interpretação do nível de risco da população ${ }^{15}$. A importância da adoção de um critério foi demonstrada por Kurth et al. ${ }^{16}$, ao relatarem que o IMC quando considerado uma variável contínua, o incremento de uma unidade no IMC, ou $1 \mathrm{~kg} / \mathrm{m}^{2}$, está associado com um aumento de $6 \%$ no risco total para derrame cerebral a partir de $23 \mathrm{~kg} / \mathrm{m}^{2}$.

O percentual de gordura $(\% \mathrm{G})$ também é um indicador utilizado para a classificação de risco em relação à obesidade. A precisão com que se pode estimar a densidade corporal e, por conseguinte, o \%G, levou inúmeros pesquisadores a se preocuparem com padrões ideais ou desejáveis para a quantidade relativa de gordura corporal. No entanto, a precisão do \% em relação ao IMC possibilita uma classificação mais precisa do estado de saúde e, dessa maneira, torna-se mais indicado para a determinação do diagnóstico de sobrepeso/obesidade. Além disso, o IMC é apenas uma escala matemática, que difere entre homens e mulheres com a mesma estatura e, inferir o mesmo grau de adiposidade e risco para saúde é falso, logo, a escala IMC comum para homens e mulheres tem sido questionada ${ }^{23}$. 
Uma das preocupações, na área da saúde, tem sido em analisar diferentes diagnósticos antropométricos de excesso de peso que sejam aplicáveis em diferentes situações, como: em estudos epidemiológicos, avaliações diárias em academias, clínicas e centros esportivos; e que permita monitorar as alterações da composição corporal com o envelhecimento.

Portanto, esse estudo teve como objetivo investigar a prevalência da obesidade e sobrepeso por dois procedimentos de diagnóstico antropométrico (IMC e \%G) em homens entre 20 e 40 anos de idade ao iniciar em programas de exercício físico em academias.

\section{Metodologia}

\section{Amostra}

Estudo de corte transversal, no qual participaram 400 sujeitos do sexo masculino, de 20 a 40 anos de idade, de cinco academias de ginástica, da cidade de Curitiba, Estado do Paraná, Brasil. Os sujeitos foram convidados a participar de forma voluntária durante a primeira avaliação antropométrica no ingresso na academia. A amostra foi dividida em quatro faixas etárias de 5 em 5 anos, ou seja, de 20-24,9; 25-29,9; 30-34,9 e 35-40 anos de idade. O número de sujeitos foi pré-determinado em 100 sujeitos para cada faixa etária, sendo adotado a saturação como critério de encerramento de coleta de dados realizada pelo autor do presente estudo com o consentimento dos indivíduos.

A classificação do estado nutricional segundo os dois diagnósticos antropométricos foi da seguinte forma: o IMC seguiu os pontos de corte da OMS19: $<18,5 \mathrm{~kg} / \mathrm{m}^{2}$, (abaixo do normal); entre 18,5-24,9 kg/m², (normal); entre 25-29,9 $\mathrm{kg} / \mathrm{m}^{2}$, (sobrepeso) e $>=30 \mathrm{~kg} / \mathrm{m}^{2}$, (obesidade). Para o \%G, seguiu-se a indicação de Lohman ${ }^{17}$, onde $\% \mathrm{G}<6$ (baixo peso); 6-15, (normal); 16-24\%, (acima da média) e $>=25 \%$ (obesidade).

\section{Procedimentos}

Para as mensurações de dobras cutâneas (DC, $\mathrm{mm})$, usaram-se as indicações de Harrison et al. ${ }^{12}$. Para a densidade corporal, foi utilizada a equação a seguinte equação ${ }^{22}$ :

$$
\begin{aligned}
& \mathrm{D}=1,10726863-0,00081201 * 4 \mathrm{DC}+ \\
& +0,00000212 * 4 \mathrm{DC} 2-0,00041761 * \mathrm{ID}
\end{aligned}
$$

Onde: 4DC é o somatório de quatro dobras cutâneas (subescapular + tríceps + supra-ilíaca oblíqua + panturrilha medial) e ID = idade em anos completos. O percentual de gordura $(\% \mathrm{G})$ foi estimado pela equação de Siri26, onde $\% \mathrm{G}=[(4,95 / \mathrm{D})-4,5] 100$. A massa corporal magra (MCM) foi calculada, $\mathrm{MCM}=(\% \mathrm{G} / 100) \mathrm{MC}$. Para o IMC, utilizou-se a fórmula, IMC= $=\mathrm{MC}(\mathrm{kg}) /$ estatura $^{2}(\mathrm{~m})$.

\section{Estatística}

Para a análise dos dados, utilizou-se a estatística descritiva, a razão de prevalência $(\mathrm{RP})$ e a análise de variância (ANOVA oneway) que é robusta a violações da homocedasticidade quando o número de observações de cada grupo é igual ou aproximadamente igual ${ }^{21}$; e o post-hoc de Scheffé $(p<0,05)$

\section{Resultados}

$\mathrm{Na}$ tabela 1 são apresentados os valores da estatística descritiva das variáveis antropométricas e da composição corporal da amostra estudada. As diferenças estatísticas significativas $(\mathrm{p}<0,05)$ foram observadas nas variáveis IMC, $\% \mathrm{G}$ e MG entre as faixas etárias, sendo que a de $34-40$ anos de idade diferiu das mais jovens. Os resultados indicam que o $\% \mathrm{G}$ dentro da classificação normal (6-14\%), foi extrapolado por todas as faixas etárias. O maior incremento no \%G ocorreu após 30 anos de idade, quando os valores médios passaram de $18,1 \%$ para $21,1 \%$, entre as faixas etárias 30-34 e 35-39 anos. 
Tabela 1: Características antropométricas e composição corporal de homens de 20 a 40 anos de idade.

\begin{tabular}{|c|c|c|c|c|c|c|}
\hline Idade & $\begin{array}{c}20-24 \text { anos } \\
(\mathrm{n}=100)\end{array}$ & $\begin{array}{c}25-29 \text { anos } \\
\quad(n=100)\end{array}$ & $\begin{array}{c}\text { 30-34 anos } \\
(\mathrm{n}=100)\end{array}$ & $\begin{array}{c}\text { 35-39 anos } \\
(\mathrm{n}=100)\end{array}$ & $\begin{array}{c}20-40 \text { anos } \\
(n=400)\end{array}$ & $p$ valor \\
\hline Variável & $S$ & $S$ & $S$ & $S$ & $S$ & \\
\hline Idade, anos & $22,5 \pm 1,5$ & $27,5 \pm 1,4$ & $32,2 \pm 1,5$ & $37,2 \pm 1,5$ & $29,9 \pm 5,7$ & \\
\hline $\mathrm{EST}, \mathrm{cm}$ & $178,0 \pm 6,9$ & $178,1 \pm 6,8$ & $178,3 \pm 6,7$ & $176,4 \pm 6,8$ & $177,7 \pm 6,8$ & 0,152 \\
\hline $\mathrm{MC}, \mathrm{kg}$ & $78,3 \pm 11,2$ & $78,6 \pm 11,9$ & $80,1 \pm 13,5$ & $82,2 \pm 13,4$ & $79,8 \pm 12,6$ & 0,102 \\
\hline $\mathrm{IMC}, \mathrm{kg} / \mathrm{m}^{2}$ & $24,7 \pm 3,2^{A}$ & $24,7 \pm 3,1^{\mathrm{A}}$ & $25,2 \pm 3,8^{A}$ & $26,5 \pm 4,4^{\mathrm{B}^{\star}}$ & $25,3 \pm 3,7$ & 0,001 \\
\hline$\% G$ & $16,0 \pm 5,1^{\mathrm{A}}$ & $16,9 \pm 5,0^{A, B}$ & $18,1 \pm 5,6^{\mathrm{B}}$ & $21,1 \pm 5,5^{\mathrm{c}^{\star}}$ & $18,0 \pm 5,6$ & 0,000 \\
\hline MG, kg & $12,9 \pm 5,8^{A}$ & $13,7 \pm 5,9^{A}$ & $15,0 \pm 7,2^{A}$ & $17,9 \pm 7,4^{\mathrm{B}^{*}}$ & $14,9 \pm 6,8$ & 0,000 \\
\hline $\mathrm{MCM}, \mathrm{kg}$ & $65,4 \pm 7,2$ & $64,9 \pm 7,6$ & $65,1 \pm 7,8$ & $64,4 \pm 7,6$ & $65,0 \pm 7,5$ & 0,787 \\
\hline
\end{tabular}

EST = estatura; $\mathrm{MC}=$ massa corporal; IMC= Índice de Massa Corporal; \%G= percentual de gordura; $\mathrm{MG}=$ massa de gordura; $\mathrm{MCM}=$ massa corporal magra.

* 20-24 anos $\ltimes 25-29$ anos, 30-34 anos e 35-39 anos.

** 30-34 anos $\star 20-24$ anos.

Quanto ao IMC (tabela 1), entre as faixas etárias de 20-24 e 25-29 anos os valores médios encontravam-se dentro de normalidade (18,5 a $24,9 \mathrm{~kg} / \mathrm{m}^{2}$ ) e que nos demais grupos etários os sujeitos foram classificados com sobrepeso (25 a $29,9 \mathrm{~kg} / \mathrm{m}^{2}$ ).

As tabelas 2 e 3 mostram as razões de prevalência de ambos os diagnósticos antropométricos de obesidade, nas diferentes faixas etárias. Optou-se por considerar o grupo com idade mais baixa (20-24 anos) como sendo aquela de referência.

Observou-se (tabela 2) que a faixa etária formada por sujeitos com idade entre 35-39 anos apresentou $300 \%$ maior prevalência de obesidade, pelo diagnóstico de $\% \mathrm{G}(>=25 \%$ ), do que o grupo de referência $\left(\mathrm{RP}=4,0 ; \mathrm{IC}_{95 \%}=1,71-9,36\right)$, sendo esta diferença significativa $(\mathrm{p}<0,05)$. Também foram observadas diferenças significativas na classificação 16-24\%. Neste caso, o risco de sobrepeso foi $45 \%$ superior para os sujeitos da faixa etária 35-39 anos do que aqueles na faixa etária de 20-24 anos (faixa etária de referência; $\mathrm{RP}=1,45 ; \mathrm{IC} 95 \%=1,11-1,90)$. Outras diferenças estatísticas significativas $(\mathrm{p}<0,05)$, também foram evidenciadas na classificação 6-15\%, quando os sujeitos das faixas etárias de 30-34 e 35-39 anos tinham menor prevalência ( $\% \mathrm{G}$ normal) $64 \%$ e $24 \%$, respectivamente, que em relação à faixa etária de referência.

No diagnóstico por IMC (tabela 3), diferenças significativas $(p<0,05)$ foram observadas somente nas classificações: "Normal" e "Sobrepeso". Os sujeitos na faixa etária entre 35-39 anos apresentaram prevalência 46\% maior de sobrepeso que aqueles do grupo de referência $\left(\mathrm{RP}=0,46 ; \mathrm{IC}_{95 \%}=1,03-2,06\right) . \mathrm{O}$ contrário aconteceu na classificação "Normal" $\left(\mathrm{IMC}=18,5-24,9 \mathrm{~kg} / \mathrm{m}^{2}\right)$, na qual a prevalência foi $64 \%$ menor $\left(\mathrm{RP}=0,64 \mathrm{IC}_{95 \%}=0,45-0,86\right)$, entre os sujeitos da faixa etária 35-39 anos, que aqueles da referência (20-24 anos de idade).

\section{Discussão}

Nesta amostra de adultos iniciantes de programas de exercício físico em academia, a prevalência de excesso de peso foi significantemente $(\mathrm{p}<0,05)$ maior no diagnóstico antropométrico de $\% \mathrm{G}(66,2 \%)$ que no de $\operatorname{IMC}(45,6 \%)$, 
Prevalência de sobrepeso e obesidade em homens adultos segundo dois critérios de diagnóstico antropométrico Ronaldo Domingues Filardo, Edio Luiz Petroski

Tabela 2: Razão de Prevalência de obesidade e Intervalo de Confiança segundo o diagnóstico antropométrico de \%G em homens.

\begin{tabular}{|c|c|c|c|c|c|c|c|c|}
\hline \multirow[b]{2}{*}{ Idade } & \multicolumn{2}{|c|}{$<6 \%$} & \multicolumn{2}{|r|}{$6-15 \%$} & \multicolumn{2}{|c|}{$16-24 \%$} & \multicolumn{2}{|r|}{ é25\% } \\
\hline & $\mathbf{N}$ & $\begin{array}{c}\mathbf{R P} \\
\left(\mathrm{IC}_{95 \%}\right)\end{array}$ & $\mathbf{N}$ & $\begin{array}{c}\mathbf{R P} \\
\left(\mathrm{IC}_{95 \%}\right)\end{array}$ & $\mathbf{N}$ & $\begin{array}{c}\mathbf{R P} \\
\left(\mathrm{IC}_{95 \%}\right)\end{array}$ & $\mathbf{N}$ & $\begin{array}{c}\mathbf{R P} \\
\left(\mathrm{IC}_{95 \%}\right)\end{array}$ \\
\hline $20-24$ & - & - & 50 & 1 & 44 & 1 & 6 & 1 \\
\hline $25-29$ & - & - & 41 & $\begin{array}{c}0,82 \\
(0,60-1,11)\end{array}$ & 49 & $\begin{array}{c}1,11 \\
(0,83-1,50)\end{array}$ & 10 & $\begin{array}{c}1,67 \\
(0,63-4,41)\end{array}$ \\
\hline $30-34$ & - & - & 32 & $\begin{array}{c}0,64 \\
(0,45-0,91)\end{array}$ & 56 & $\begin{array}{c}1,27 \\
(0,96-1,69)\end{array}$ & 12 & $\begin{array}{c}2,0 \\
(0,78-5,12)\end{array}$ \\
\hline $35-39$ & - & - & 12 & $\begin{array}{c}0,24 \\
(0,14-0,42)\end{array}$ & 64 & $\begin{array}{c}1,45^{\star} \\
(1,11-1,90)\end{array}$ & 24 & $\begin{array}{c}4,0^{*} \\
(1,71-9,36)\end{array}$ \\
\hline $\begin{array}{l}\text { Prevalência } \\
\text { de obesidade }\end{array}$ & - & - & 33,8 & & 53,2 & & $13 \%$ & \\
\hline
\end{tabular}

$\mathrm{RP}=$ Razão de Prevalência; $\mathrm{IC}=$ Intervalo de confiança; * diferença significativa $\mathrm{p}<0,05$.

Tabela 3: Razão de Prevalência de obesidade e Intervalo de Confiança segundo o diagnóstico antropométrico de IMC em homens.

\begin{tabular}{|c|c|c|c|c|c|c|c|c|}
\hline \multirow[b]{2}{*}{ Idade } & \multicolumn{2}{|r|}{$<6 \%$} & \multicolumn{2}{|c|}{$6-15 \%$} & \multicolumn{2}{|c|}{$16-24 \%$} & \multicolumn{2}{|r|}{ é25\% } \\
\hline & $\mathbf{N}$ & $\begin{array}{c}\mathbf{R P} \\
\left(\mathrm{IC}_{95 \%}\right)\end{array}$ & $\mathbf{N}$ & $\begin{array}{c}\mathbf{R P} \\
\left(\mathrm{IC}_{95 \%}\right)\end{array}$ & $\mathbf{N}$ & $\begin{array}{c}\mathbf{R P} \\
\left(\mathrm{IC}_{95 \%}\right)\end{array}$ & $\mathbf{N}$ & $\begin{array}{c}\mathbf{R P} \\
\left(\mathrm{IC}_{95 \%}\right)\end{array}$ \\
\hline $20-24$ & 2 & 1 & 58 & 1 & 33 & 1 & 7 & 1 \\
\hline $25-29$ & - & - & 64 & $\begin{array}{c}1,11 \\
(0,86-1,39)\end{array}$ & 30 & $\begin{array}{c}0,91 \\
(0,60-1,97)\end{array}$ & 6 & $\begin{array}{c}0,86 \\
(0,39-2,46)\end{array}$ \\
\hline $30-34$ & 1 & $\begin{array}{c}0,50 \\
(0,15-2,44)\end{array}$ & 56 & $\begin{array}{c}0,97 \\
(0,76-1,26)\end{array}$ & 34 & $\begin{array}{c}1,04 \\
(0,70-1,52)\end{array}$ & 9 & $\begin{array}{c}1,29 \\
(0,50-3,32)\end{array}$ \\
\hline $35-39$ & - & - & 37 & $\begin{array}{c}0,64 \\
(0,45-0,86)\end{array}$ & 48 & $\begin{array}{c}1,46^{\star} \\
(1,03-2,60)\end{array}$ & 15 & $\begin{array}{c}2,15 \\
(0,91-5,03)\end{array}$ \\
\hline $\begin{array}{l}\text { Prevalência } \\
\text { de obesidade }\end{array}$ & 1,5 & - & 53,6 & & 36,3 & & 9,3 & \\
\hline
\end{tabular}

$\mathrm{RP}=$ Razão de Prevalência; IC= Intervalo de confiança; * diferença significativa $p<0,05$.

sendo $53,2 \%$ para sobrepeso e $13 \%$ para obesidade. Já no diagnóstico de IMC, a prevalência de sobrepeso e obesidade foi $36,3 \%$ e $9,3 \%$, respectivamente. Recentemente, Cordeiro ${ }^{5}$ comparou a prevalência de obesidade em homens e mulheres idosas. Quando o autor estimou o \%G
(DEXA) verificou uma prevalência de $32 \%$, enquanto, pela classificação do IMC $\left(>30 \mathrm{~kg} / \mathrm{m}^{2}\right)$, a mesma era somente $12 \%$. Para as mulheres, a prevalência de obesidade estimada por $\% \mathrm{G}$, foi de $95,8 \%$, contra apenas $27,7 \%$ estimada pelo $\%$ G (DEXA). 
Após 35 anos de idade, em relação à faixa etária 20-24, as alterações em IMC de $1,8 \mathrm{~kg} / \mathrm{m}^{2}$, $5,1 \%$ e $M G$ na ordem de $5 \mathrm{~kg}$ chamam a atenção, pois em uma década ocorreram incrementos significativos nos indicadores de gordura corporal. No entanto, a MCM não sofreu alterações, o que vem indicar que a variação de 3,9 $\mathrm{kg}$ na $\mathrm{MC}$ e $1 \mathrm{~kg}$ na MCM, mesmo não sendo significativas, indicaram a necessidade de cuidados nessa fase de transformações corporais, principalmente no que diz respeito ao ganho da gordura corporal. Por sua vez, esse ganho da gordura corporal com o avanço da idade é marcado pela MC e \%G e traz consequentes alterações na composição corporal.

Os valores médios de \%G no presente estudo não representariam fortes riscos para a saúde, mas acima de $25 \% \mathrm{G}$ já seriam considerados de risco para doenças associadas à obesidade ${ }^{23}$. Por sua vez, Lopes et al. ${ }^{18}$ relataram que entre 20-29,9 e 30-39,9 anos há um acréscimo de 4,8\%G com o avanço da idade. Em outro estudo ${ }^{7}$ foi observado um incremento de 4,3 no $\% \mathrm{G}$ nas mesmas faixas etárias. Fermino et al. ${ }^{6}$ verificaram que o incremento na gordura corporal de $3 \%$ por década, ou seja, a média do $\% \mathrm{G}$ de 18 para $24 \%$ entre 20 e 50 anos de idade. Dessa maneira, por serem todos estes estudos com amostras brasileiras e principalmente por utilizarem a mesma equação para estimativa da densidade corporal e $\% \mathrm{G}$, pode-se inferir que é natural com o aumento da idade cronológica um acréscimo médio da ordem de $4 \% \mathrm{G}$ entre 20 e 40 anos de idade.

Quando analisada a RP entre dois critérios (\%G e IMC), foi observada no diagnóstico de $\% \mathrm{G}$ maior prevalência de sobrepeso que no IMC. Deve-se ressaltar que o $\% \mathrm{G}$ considera a composição corporal dos indivíduos, o que indicou maior possibilidade de diagnóstico de excesso de peso para faixas etárias mais velhas. A maior precisão de classificação de sujeitos obesos ou não-obesos depende da qualidade do diagnóstico, quando maior a sensibilidade e a especificidade deste, maior é a probabilidade de classificar sujeitos corretamente. Estudos recentes mostram que a classificação do IMC utilizada no presente estudo não é adequada para sujeitos em idades mais avançadas ${ }^{26}$. A não adequação é atribuída às modificações corporais, como: perda da massa livre de gordura, aumento da gordura corporal, diminuição da estatura e da água corporal total, que ocorrem com o envelhecimento ${ }^{24}$. Assim, é possível que duas pessoas com o mesmo IMC tenham diferentes percentuais de gordura corporal.

Quando analisada a prevalência de sobrepeso (sobrepeso + obesidade), através do diagnóstico de $\% \mathrm{G}$, observou-se que a mesma aumentou consideravelmente dos 20 aos 40 anos de idade, ou seja, $50 \%, 59 \%, 68 \%$ e $88 \%$, enquanto que para o diagnóstico de IMC, foi $40 \%, 36 \%, 43 \%$ e 63\%, para as faixas etárias de 20-24, 25-29, 30-34 e 35-39 anos de idade, respectivamente.

Nos EUA ${ }^{8}$, a prevalência de obesidade (IMCé30 $\mathrm{kg} / \mathrm{m}^{2}$ ) em adultos com mais de 20 anos, entre 1994-2000, atingiu 30,5\%, enquanto atingia 22,9\% entre 1988-1994. Para homens nas faixas etárias de 20-39 e 40-59 anos, a prevalência foi de $27,5 \%$ e $28,8 \%$, respectivamente. No Canadá ${ }^{28}$, a prevalência de sobrepeso em homens de 20-64 anos atingiu 60\%, em 1996, e a obesidade $14 \%$.

No Brasil, cerca de $32 \%$ da população apresentaram algum excesso de peso (IMC maior ou igual a $25 \mathrm{~kg} / \mathrm{m}^{2}$ ), sendo que homens representavam aproximadamente $27 \%$ da população $\mathrm{O}^{4}$. A obesidade entre adultos varia consideravelmente de acordo com a região e urbanização do país. Nas regiões Nordeste e Sudeste, a prevalência de sobrepeso atinge, entre 20 e 29 anos, $22,3 \%$ e a obesidade $3,4 \%$. Entre 30 e 29,9 anos, o sobrepeso, $32,2 \%$ e a obesidade, $7,8 \%$. Foi verificado que a prevalência de sobrepeso e a de obesidade, respectivamente, oscila entre $32,2 \%$ e $35,8 \%$, entre 30 e 69 anos de idade ${ }^{1}$. 
Para o IMC maior que $30 \mathrm{~kg} / \mathrm{m}^{2}$, um estudo com 455 homens da zona urbana da cidade de Pelotas (RS), entre 20 e 69 anos de idade, relatou uma prevalência de 15\% no geral, sendo que a proporção de obesidade foi crescente com a idade ${ }^{11}$. A prevalência de obesidade é preocupante devido ao relacionamento com doenças. Em um recente estudo realizado no Rio de Janeiro foi observado maior prevalência de hipertensão arterial sistêmica, dislipidemias e diabetes mellitus em indivíduos com obesidade, quando comparados aos eutróficos ${ }^{27}$.

Esses resultados sugerem que o diagnóstico por $\% \mathrm{G}$ é mais permissivo para classificar sobrepeso em homens adultos jovens e como tal, aprimora a prescrição nutricional e da atividade física; bem como, permite a observação do quanto a composição corporal sofre alterações entre diferentes faixas etárias.

Mesmo que o presente estudo tenha algumas limitações, como a falta de controle do nível socioeconômico, de um padrão-ouro de diagnóstico de obesidade, o fato da composição da amostra ser formada por sujeitos que ingressaram em academias de ginástica, os resultados sugerem que as prevalências de excesso de peso são diferentes entre os diagnósticos antropométricos. Estas prevalências são maiores quando estimadas por meio do \%G. Em estudos populacionais, o IMC é sem dúvida uma medida útil na triagem da obesidade devido à facilidade de obtenção dos dados, interpretação dos resultados e, devido à sua relação com riscos à saúde. $\mathrm{O}$ indicador antropométrico de $\% \mathrm{G}$, embora tenha menor praticabilidade, poderia ser facilmente utilizado em academia de ginástica, pois a mesma conta com pessoal capacitado para realizar as mensurações e elaborar relatórios. Acredita-se que este procedimento é mais uma ferramenta disponível a ser utilizada na prevenção da obesidade, pois salienta a necessidade de uma intervenção nutricional; bem como, permite a observação do quanto a composição corporal sofre alterações com o envelhecimento.

De acordo com os dados apresentados, pode-se concluir que ocorre um incremento significativo da gordura corporal dos 20 aos 40 anos de idade, enquanto a massa corporal magra permanece constante.

$\mathrm{O}$ estudo encontrou alta prevalência de excesso de peso (um a cada três sujeitos) ao se inscrever em academia de ginástica.

O risco de obesidade pode se melhor identificado quando o excesso de peso é diagnosticado através do \%G.

Finalmente, é recomendável a indicação da classificação do IMC para estudos populacionais e do $\% \mathrm{G}$ para academias como um recurso mais refinado para a estimativa da composição corporal, para a indicação de peso ideal em relação à quantidade de gordura corporal total.

\section{Correspondência}

Rua Francisco Xavier Oliveira, 525

Curitiba / Paraná, Brasil

Cep: $81480-070$

Telefone: (41) 9102-6435

E-mail: ronaldofilardo@yahoo.com.br

E-mail: petrosky@cds.ufsc.br 


\section{Referências}

1. Abrantes, M. M.; Lamounier, J. A.M; Colosimo, E. A. (2003). Prevalência de sobrepeso e obesidade nas regiões Nordeste e Sudeste do Brasil. Rev Assoc Med Bras 49(2) :162-6.

2. Batista Filho, M.; Rissin, A. A. (2003). Transição nutricional no Brasil: tendências regionais e temporais. Cad Saúde Pública 19: S181-S191.

3. Caterson, I. D.; Gil, T. P. (2002). Obesity: epidemiology and possible prevention. J Clin Endocrinol Metab 16(4): 595-610.

4. Coitinho, D. C.; Leão, M. M.; Recine, E.; Sichieri, R. (1991). Condições nutricionais da população brasileira: adultos e idosos (Pesquisa Nacional sobre Saúde e Nutrição). Brasília: Ministério da Saúde - INAN.

5. Cordeiro, B. A. (2006). Sensibilidade e especificidade do indice de massa corporal (IMC) no diagnóstico de obesidade em idosos: comparaşão com a absortometria por raio-x de dupla energia (DEXA) proposta de novos pontos de corte. (Dissertação): Universidade Federal de Santa Catarina.

6. Fermino, R.; Filardo, R. D.; Pires-Neto, C. S. (2003). Valores antropométricos e da composição corporal de homens entre 20 e 60 anos de idade. In: Anais do XXVI Simpósio Internacional de Ciências do Esporte - Atividade Física Construindo Saúde. São Paulo: Brasil, 189.

7. Filardo, R. D.; Pires-Neto, C. S. (2001). Indicadores antropométricos e da composição corporal de homens e mulheres entre 20 e 39,9 anos de idade. Rev Bras Cineantropom Desempenho Hum 3(1): 55-62.

8. Flegal, K. M.; Carrol, M. D.; Ogden, Cl.; Johnson, Cl. (2002). Prevalence and trends in obesity among US Adults, 1999-2000. JAMA 288: : 1723-7.

9. Gaesser, G. A. (1999). Thinness and weight loss: beneficial or detrimental to longevity? Med Sci Sports Exer 31: 1118-28.

10. Garn, S. M.; Leonard, W. R.; Hawthorne , V. M. (1997). Three limitations of the body mass index. Am J Clin Nutr 44: 996-7.
11. Gigante, D. P.; Barros, F. C.; Post, C. L. A.; Olinto, M. T. A. (1997). Prevalência de obesidade em adultos e seus fatores de risco. Rev Saúde Pública 31(3): 236-46.

12. Harrison, G. G.; Buskirk, E. R.; Carter , J. E. L.; Johnston, F. E.; Lohman, T. G.; Pollock, M. L.; Roche, A. F.; Wilmore, J. (1998); Skinfold thickness and measurement technique. In: Lohman, T. G.; Roche, A. F.; Martorell, R. Anthropometric standardization reference manual. Champaign: Human Kinetics Publishers, 55-70.

13. Hubbard, V. S. (2000). Defining overweight and obesity: what are the issues? Am J Clin Nutr 72: 1067-8.

14. Katzmarzyk, P. T.; Pérusse, L.; Malina, R. M.; Bouchard, C. (1999). Seven-year stability of indicators of obesity and adipose tissue distribution in the Canadian population. Am J Clin Nutr 69: 1123-9.

15. Kuczmarski, R. J.; Flegal, K. M. (2000). Criteria for definition of overweight in transition: background and recommendations for the United States. Am J Clin Nutr 72: 1074-81.

16. Kurth, T.; Gaziano, M.; Berger, K.; Kase, C. S.; Rexrode, K. M.; Cook, N. R.; Buring, J. E.; Manson, J. E. (2002). Body Mass Index and risk of stroke in men. Arch Intern Med 162: 2557-62.

17. Lohman, T. G. (1992). Advances in body composition assessment. Champaign: Human Kinetics Publishers.

18. Lopes, A. S.; Nahas, M. V.; Duarte, M. F. S.; Pires-Neto, C. S. (1995). Distribuição da gordura corpórea subcutânea e índices de adiposidade em indivíduos de 20 a 67 anos de idade. Rev Bras Ativ Fis Saúde 1(2): 15-26.

19. Organisation Mondiale de la Sante.(2000). Obesity: Preventing and Managing the Global Epidemic: Report of a WHO Consultation on Obesity. Genève: World Health Organization.

20. Passos, V. M. A.; Barreto, S. M.; Diniz, L. M.; Lima-Costa, M. F. (2005) Type 2 diabetes: 
prevalence and associated factores in a brazilian community - the Bambuí health and aging study. São Paulo. Med J 123(2): 66-71.

21. Pestana, M. H.; Gageiro, J. N. (2000) Análise de dados para ciências sociais: a complementariedade do SPSS. Editora Silabo.

22. Petroski, E. L. (1995). Desenvolvimento e validação de equações generalizadas para a estimativa da densidade corporal em adultos [tese]. Santa Maria: Universidade Federal de Santa Maria.

23. Ross, W. D. (1997). Agrupando amostras que diferem alometricamente em tamanho. Rev Bras Med Esporte 96: 95-100

24. Sardinha, L. B.; Texeira, P. J. (2000). Obesity screning in older women with body mass index: a receiver operating characteristic (ROC) analysis. Sci Sports 15: 212-219.

25. Seidell, J. C.; Flegal, K. M. (1997). Assessing obesity: classification and epidemiology. $\mathrm{Br} \mathrm{Med}$ Bull 53(2): 238-52.

26. Siri, W. E. (1961). Body composition from fluid space and density. In: Brozek, J., Hanschel, A., editors. Techniques for measuring body composition.
Washington DC: National Academy of Science, 223-244.

27. Souza, L. J.; Gicovate-Neto, C.; Chalita , F. E. B.; Reis, A. F. F.; Bastos, D. A.; Souto-Filho, J. T. D.; Souza, T. F.; Côrtes, V. A. (2003). Prevalência de obesidade e fatores de risco cardiovascular em campo, Rio de Janeiro. Arq Bras Endocrinol Metab 47(6): 669-76.

28. Tremblay, M. S.; Katzmarzyk, P. T.; Williams, J. D. (2002). Temporal trends in overweight and obesity in Canada, 1981-1996. Int J Obes 26: 538-43.

29. World Health Organization (2004). Obesity and overweight.

http://www.who.int/hpr/NPH/docs/gs_ob esity.pdf. Acessado em 02/03/2004

30. World Health Organization (WHO) (1995). Expert Committee on Physical Status: the Use and Interpretation of Anthropometry Physical status: the use and interpretation of anthropometry: report of a WHO expert committee. WHO technical. 